\title{
Physiopathologie de la mucoviscidose
}

\author{
Scott H. Donaldson Richard C. Boucher \\ Centre de traitement et d'étude de la mucoviscidose de l'Université de Caroline du Nord, \\ Chapel Hill, N.C., États-Unis
}

\section{Mots-clés}

Mucoviscidose $\cdot$ CFTR · Canal sodique épithélial · Clairance mucociliaire $\cdot$ Transport ionique $\cdot$ Surfactant bronchique

\section{Résumé}

La compréhension des modalités de survenue de l'atteinte respiratoire due à la mucoviscidose est un sujet de recherche depuis plusieurs décennies. Le clonage du gène régulateur de la conductance transmembranaire (CFTR) et d'autres progrès dans le domaine de la biologie de l'épithélium bronchique ont permis de beaucoup mieux connaître la physiopathologie de la maladie. Les meilleures données disponibles indiquent que l'anomalie sous-jacente est une dysrégulation du transport ionique qui induit une déplétion en liquide de la surface bronchique, donc une déshydratation, et une altération associée de l'élimination du mucus. La stase du mucus prédispose alors le poumon mucoviscidosique à une infection bactérienne qui devient rapidement chronique en raison de la nature des sécrétions. La réponse inflammatoire neutrophile persistante à cette infection chronique provoque alors des lésions bronchiques progressives et permanentes et, de ce fait, des bronchectasies et une insuffisance respiratoire sont habituelles quand l'atteinte respiratoire mucoviscidosique atteint le stade terminal. Le développement de nouveaux traitements ciblant l'anomalie sous-jacente de la déshydratation de la surface des voies aériennes permet d'espérer qu'il sera possible de prévenir cette cascade d'événements.

Copyright @ 2006 Nestec Ltd., Vevey/S. Karger AG, Basel

\section{Introduction}

La mucoviscidose est la maladie génétique fatale la plus fréquente dans les populations caucasiennes. Des mutations du gène régulateur de la conductance transmembranaire (CFTR), situé sur le bras long du chromosome 7 , sont responsables d'une maladie autosomique récessive. A ce jour, plus de 1400 mutations individuelles ont été identifiées et enregistrées dans la base de données sur les mutations associées à la mucoviscidose (Cystic Fibrosis Mutation Database; http://www.genet.sickkids. on.ca/cftr/), ce qui rend impraticable le dépistage de masse par des techniques uniquement génétiques. L’allèle mutant $\Delta$ F508, qui code pour la délétion d'un seul acide aminé dans cette protéine qui en compte 1480 , est de loin l'allèle le plus prévalent, représentant $66 \%$ des mutations au plan mondial [1]. Le phénotype classique de la mucoviscidose est très complexe, impliquant plusieurs organes à revêtement épithélial [De Boeck, pp. 124-126], bien que les manifestations respiratoires soient la principale source de morbidité et de mortalité. Ces dernières années, des progrès significatifs ont été accomplis vers une meilleure connaissance de la voie qui relie les mutations du gène CFTR aux manifestations cliniques de la mucoviscidose et, en particulier, des mécanismes qui sous-tendent la défaillance apparente des défenses pulmonaires. Dans la présente analyse, nous nous centrerons sur les mécanismes physiopathologiques qui rendent le poumon mucoviscidosique vulnérable à la survenue d'infections bactériennes chroniques et aux événements consécutifs responsables de bronchectasies progressives.

$\begin{array}{ll}\text { KARGER } & \text { ( 2006 Nestec Ltd., Vevey/S. Karger AG, Basel } \\ \text { Fax }+41613061234 & \text { 0250-9644/06/0643-0101\$23.50/0 } \\ \begin{array}{l}\text { E-Mail karger@karger.ch } \\ \text { www.karger.com }\end{array} & \begin{array}{l}\text { Accessible en ligne à: } \\ \text { www.karger.com/anf }\end{array}\end{array}$

Scott H. Donaldson, MD

6019 Thurston Bowles Building, CB\# 7248

University of North Carolina at Chapel Hill

Chapel Hill, NC 27599 (USA)

Tel. +1 919966 9198, Fax +1 919966 5178, E-Mail Scott_Donaldson@med.unc.edu 
Le CFTR est un régulateur du transport épithélial du sel et de l'eau

Les premières notions indiquant que la mucoviscidose résulte d'une anomalie du transport des électrolytes provenaient d'observations indiquant que la sueur des sujets atteints contenait une concentration élevée de sodium et de chlorure [2,3]. Plusieurs études ont apporté des données supplémentaires démontrant une anomalie du transport ionique dans divers organes affectés, dont les bronches [4], les canaux pancréatiques [5], les canaux biliaires $[6,7]$ et le côlon [8]. Le clonage du gène CFTR $[9,10]$ et l'identification consécutive de sa nature de canal chlorure $[11,12]$ ont été des progrès majeurs qui ont confirmé le lien entre la dysrégulation du transport ionique et les diverses manifestations viscérales de la mucoviscidose. Il est toutefois établi aujourd'hui que le rôle du CFTR dans la physiopathologie épithéliale s'étend au-delà de sa fonction de canal $\mathrm{Cl}^{-}$, car de nombreuses études ont démontré qu'il intervenait dans la régulation d'autres canaux ioniques. Il faut noter en particulier que le CFTR exerce un effet inhibiteur sur le canal sodique épithélial ( $\mathrm{ENaC})[13$, 14] par un mécanisme encore indéterminé, tout en influençant également l'activité d'autres canaux chlorure distincts $[15,16]$. Le CFTR est donc positionné comme un régulateur central du transport du sel et de l'eau à travers de nombreux épithéliums, et son absence entraîne des anomalies du transport ionique affectant spécifiquement certains organes. En conséquence, une réduction de la sécrétion liquidienne nette à travers l'épithélium atteint est un point commun qui aboutit à des obstructions canalaires (pancréas, voies biliaires, bronchioles, canaux déférents) et aux dysfonctions viscérales consécutives (insuffisance pancréatique exocrine, cirrhose, bronchectasies, infertilité masculine).

\section{Physiopathologie de l'atteinte respiratoire due à la mucoviscidose}

L'atteinte respiratoire due à la mucoviscidose diffère des autres manifestations viscérales de cette maladie car (1) elle est la cause du décès prématuré d'environ $95 \%$ des patients et (2) un phénotype infectieux chronique apparaît uniquement dans les poumons, avec une intense réponse inflammatoire associée. Il est donc justifié de discuter de façon plus approfondie les processus physiopathologiques qui contribuent à cette atteinte respiratoire.

Les données actuelles suggèrent que le poumon mucoviscidosique n'est pas infecté ni inflammatoire au mo- ment de la naissance $[17,18]$. Cependant, en quelques mois ou années, les stigmates de l'infection, d'abord récidivante puis chronique, commencent à apparaître. Les études microbiologiques révèlent une évolution plutôt typique des agents pathogènes, des virus respiratoires, Haemophilus influenzae et Staphylococcus aureus, prédominant au début de la vie. Avec le temps, des agents pathogènes plus nocifs et de plus en plus résistants, dont Pseudomonas aeruginosa et d'autres bactéries Gram négatif (par exemple complexe Burkholderia cepacia, Stenotrophomonas maltophilia, Achromobacter xylosoxidans), dominent souvent le tableau clinique. La normalité des tests directs de l'immunité systémique et l'absence de phénotype infectieux en dehors des voies respiratoires suggère qu'une anomalie locale des défenses pulmonaires est responsable de la survenue de l'atteinte respiratoire chronique due à la mucoviscidose. De fait, l'intense réponse inflammatoire neutrophile aux infections bronchiques parait plus puissante et persistante qu'en l'absence de mucoviscidose [19-22], mais le poumon mucoviscidosique ne parvient finalement plus à éliminer les agents pathogènes quand ceux-ci se sont établis. Cette anomalie des défenses innées des voies aériennes a fait l'objet de recherches intensives, et est la cible récente de traitements visant à prévenir ou ralentir la cascade d'événements pathogènes qui amènent ultimement à la destruction progressive des poumons.

\section{Les mécanismes innés de défense des poumons normaux}

Le poumon est continuellement exposé à des agents nocifs et infectieux, et l'évolution l'a donc doté de plusieurs lignes de défense assurant l'assainissement permanent des voies aériennes sans susciter une réponse inflammatoire potentiellement nuisible. Le système de clairance mucociliaire paraît être l'élément capital des défenses des voies aériennes et est le siège d'anomalies qui aboutissent à des affections respiratoires génétiques telles que la mucoviscidose et la dyskinésie ciliaire primitive. D’autres éléments importants de ce système de défense sont les leucocytes résidant localement (par exemple macrophages alvéolaires et bronchiques), les immunoglobulines de la muqueuse et les composés antimicrobiens secrétés (par exemple lysozyme, lactoferrine), qui tous peuvent neutraliser les micro-organismes qui échappent à la première ligne de défense, la clairance mucociliaire.

Le fonctionnement normal de cette clairance nécessite des activités coordonnées de sécrétion de mucus, de 
transport de $\mathrm{NaCl}$ et d'eau et de battement ciliaire. La sécrétion de mucus crée une couverture protectrice qui fixe efficacement les particules inhalées par l'intermédiaire de sa panoplie d'épitopes constitués d'hydrates de carbone, où elles sont piégées par un flux turbulent. La couche muqueuse, qui flotte au-dessus d'une couche liquide moins visqueuse et physiquement distincte, est propulsée vers le haut par l'association des battements ciliaires coordonnés, du débit aérien et de la toux. La couche liquide sous-jacente, souvent appelée «sol» ou «liquide périciliaire» (LPC) est elle-même très complexe et spécifiquement structurée afin de procurer un environnement ne freinant pas le battement ciliaire tout en permettant un couplage mécanique efficace entre l'extrémité des cils et la couche de mucus. Le transport ionique actif crée la force motrice de la sécrétion et de l'absorption régulées de liquide [23], ce qui maintient le LPC à une hauteur adéquate (environ un huitième de la longueur d'un cil totalement étiré) et une hydratation adéquate de la couche muqueuse [24]. De façon peut-être plus importante, le LPC peut également fonctionner comme un lubrifiant évitant que la couche muqueuse n'adhère aux surfaces cellulaires, ce qui facilite l'élimination du mucus sous l'effet des battements ciliaires comme sous celui de la toux [25].

\section{L'anomalie des défenses innées de l'hôte dans la mucoviscidose}

Diverses hypothèses ont été avancées afin d'expliquer l'association d'un excès de mucus bronchique, d'infections bactériennes typiques (particulièrement à $P$. aeruginosa) et d'une inflammation neutrophile intense qui est caractéristique de l'atteinte respiratoire due à la mucoviscidose. L'accroissement de la fixation de bactéries dans les bronches mucoviscidosiques, dû à une altération de la glycosylation des protéines luminales [26-28], et la réduction de la fixation, de l'internalisation et de l'élimination de Pseudomonas [29, 30], qui sont médiées par le CFTR, ont été toutes deux proposées à titre de facteurs prédisposant les patients atteints d'une mucoviscidose à une infection bronchique chronique. Aucune de ces deux hypothèses n'a paru toutefois expliquer totalement l'atteinte respiratoire due à la mucoviscidose. L'hypothèse «altération de la glycosylation» n'explique pas l'infection des patients ayant un génotype autre que $\Delta \mathrm{F} 508 / \Delta \mathrm{F} 508$ [31]. La seconde hypothèse, selon laquelle le CFTR agirait comme un récepteur de Pseudomonas, n'explique pas l'apparition d'une atteinte respiratoire cliniquement ma-

Physiopathologie de la mucoviscidose nifeste avant toute infection due à $P$. aeruginosa. De plus, les patients présentant des mutations de CFTR qui aboutissent à l'expression de canaux à la membrane apicale (mutations de «classe $I V »)$ ont également un phénotype infectieux, malgré la présence d'un canal CFTR peu conducteur mais autrement intact.

D'autres explications de la prédisposition inhabituelle aux infections respiratoires dans la mucoviscidose ont été centrées sur une hypothèse selon laquelle la composition des sécrétions elles-mêmes serait anormale. Les premières études ont indiqué que le liquide de surface des voies aériennes (LSVA) des patients atteints d'une mucoviscidose était relativement hypertonique (par rapport au LSVA normal) et constituait en conséquence un environnement peu adéquat pour la fonction des défensines, peptides antimicrobiens sensibles au $\mathrm{NaCl}[32,33]$. Ces études ont toutefois été largement rejetées, car les résultats d'autres travaux bien conduits ont uniformément démontré l'isotonie du LSVA (normal et mucoviscidosique) [34-38] et l'absence d'altération des molécules antimicrobiennes dominantes (par exemple lactoferrine, lysozyme) dans la mucoviscidose [39]. Une hypothèse "composite» additionnelle proposée afin d'expliquer l'altération des défenses de l'hôte dans la mucoviscidose a trait à l'absence de sécrétion de bicarbonates à travers le CFTR. Certaines études indiquent que le $\mathrm{pH}$ des sécrétions de la surface des voies aériennes pourrait être plus bas dans la mucoviscidose [40-42], mais le ou les mécanismes précis par lesquels ce phénomène peut aboutir à une infection respiratoire sont mal élucidés.

En opposition à ces hypothèses de «biologie cellulaire» et de "composition du LSVA», celle actuellement en faveur, qui repose sur le faible volume du LSVA, relie directement les anomalies précédemment décrites du transport ionique à l'altération de l'élimination du mucus. La mucoviscidose est la conséquence de l'absence de deux fonctions du CFTR dans les voies aériennes. Tout d'abord, l'influence inhibitrice médiée par le CFTR sur l'ENaC est absente. En conséquence, le sodium est absorbé de façon incontrôlée quel que soit le statut du volume du LSVA. Deuxièmement, la capacité du CFTR à susciter une sécrétion de $\mathrm{Cl}^{-}$en réponse à une diminution du volume du LSVA est également absente. De ce fait, alors que l'épithélium bronchique normal absorbe du sodium et sécrète des chlorures en réponse à la concentration de l'adénosine à la surface des voies aériennes (par l'intermédiaire des récepteurs $A_{2 b}$ qui lient l'adénosine et régulent le taux d'AMPc) afin de contrôler la quantité de LSVA [43], l'épithélium mucoviscidosique n'y parvient pas de façon efficace [44]. Au contraire, l'épithélium bronchique étant

Ann Nestlé [Fr] 2006;64:101-109 


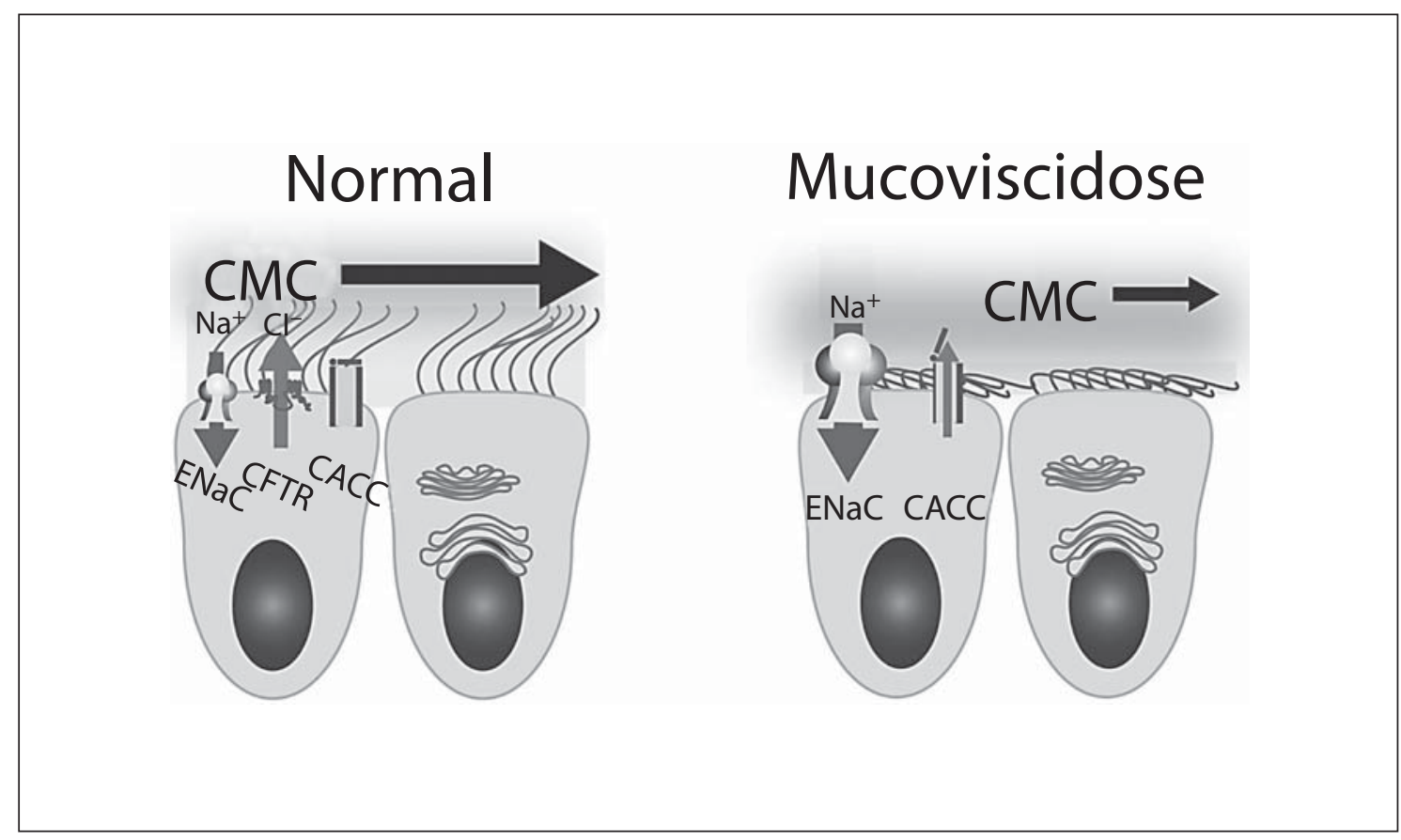

Fig. 1. Les cellules de l'épithélium respiratoire normal régulent le transport de sodium (par l'intermédiaire de l'ENaC) et de chlorure (par l'intermédiaire du CFTR et du CACC) afin de maintenir une hauteur optimale de la couche liquide périciliaire et de conforter ainsi le mouvement ciliaire tout en hydratant adéquatement la couche sus-jacentes de mucus, ce qui permet le maintien de la clairance mucociliaire (CMC). Dans la mucoviscidose, le mécanisme de transport épithélial du chlorure est absent et le sodium est hyperabsorbé (par l'intermédiaire de l'ENaC). Malgré une compensation partielle par une sécrétion de chlorure à travers le CACC, il en résulte une diminution de la couche de LPC et une déshydratation du mucus, ce qui ralentit la clairance mucociliaire.

perméable à l'eau, la combinaison d'une hyperabsorption du sodium et d'une sécrétion inadéquate de chlorure se traduit par un moindre volume de LSVA isotonique. En raison de cette réduction de volume, le LPC devient trop peu profond pour permettre des mouvements ciliaires normaux, et ce mécanisme d'élimination ne fonctionne donc pas (fig. 1). De plus, la raréfaction du LPC peut permettre un contact entre la couche muqueuse sus-jacente et les surfaces cellulaires, autorisant la survenue d'interactions adhésives entre les mucines (liées) de ces surfaces et des composants sécrétés du mucus. En conséquence, l'élimination du mucus dépendante des cils comme de la toux est altérée. Enfin, la déshydratation de la couche de mucus accroît la viscoélasticité de celle-ci, ce qui réduit sa transportabilité. La concentration élevée des mucines dans la couche de mucus resserre également les mailles à travers lesquelles les neutrophiles recrutés doivent migrer pour atteindre les bactéries intraluminales, ce qui affaiblit encore les défenses de l'hôte [45]. Cette série d'événements constitue ainsi la base physiopatholo- gique du déclenchement de l'atteinte respiratoire dans la mucoviscidose et entraîne une stase du mucus et la formation de plaques adhérentes de mucus sur les surfaces des voies aériennes. Ce sont ces plaques qui procurent l'environnement dans lequel des bactéries spécifiques peuvent persister et provoquer une bronchopathie chronique suppurée (fig. 2).

En raison de l'importance de la clairance mucociliaire pour les défenses pulmonaires, il n'est pas surprenant qu'il existe une voie redondante de sécrétion de liquide dans les voies aériennes - les canaux chlorure activés par le calcium (CACC), qui sont activés par la liaison de l'adénosine 5 '-triphosphate (ATP) aux récepteurs $\mathrm{P}_{2} \mathrm{Y}_{2}$. L'ATP est libéré par les cellules épithéliales, le plus notablement en réponse à des stimuli physiques tels que la force modérée de cisaillement due au débit aérien lors de l'inspiration et de la toux [44]. Des études récentes suggèrent que ce système bronchique est fonctionnel dans la mucoviscidose et peut procurer une hydratation de la surface des voies aériennes suffisante pour la clairance mucoci- 


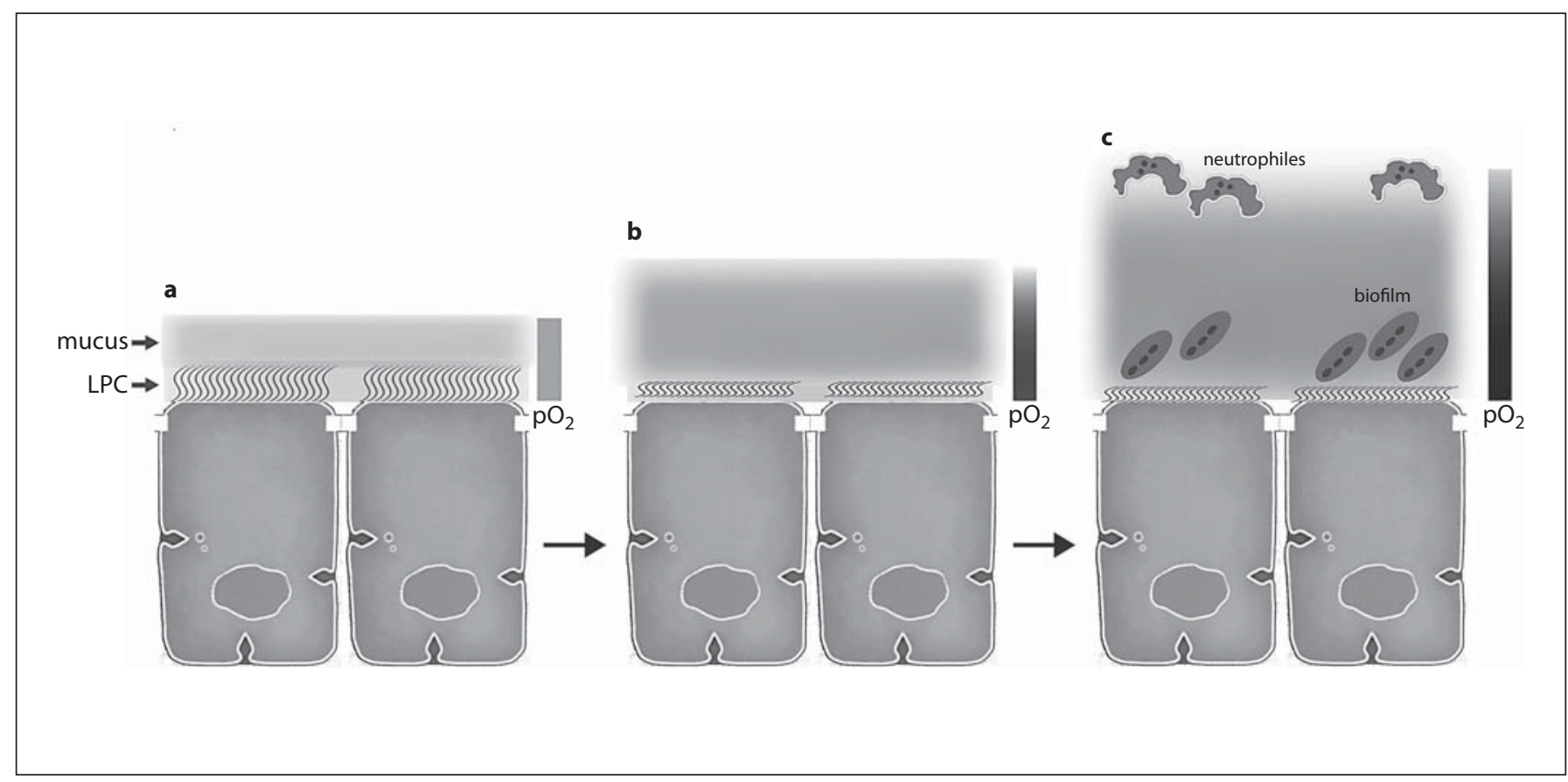

Fig. 2. a Le poumon normal entretient une couche de LPC dont la hauteur est environ le huitième de celle d'un cil totalement étiré et qui maintient l'hydratation normale de la couche de mucus. Les sécrétions de la surface des bronches sont aérobies. b Précocement dans l'atteinte respiratoire due à la mucoviscidose, des anomalies du transport ionique entraînent une réduction de la couche de LPC, ce qui s'oppose à des mouvements ciliaires normaux et amène le mucus qui s'épaissit à commencer à empiéter sur les cils. c Quand l'atteinte respiratoire est avancée, une masse très déshydratée et anaérobie de mucus s'accumule dans la lumière des bronches, adhère à la surface de celles-ci en raison de la disparition de la couche de LPC et favorise la prolifération de microcolonies de Pseudomonas dans une structure en biofilm. Les neutrophiles recrutés ne peuvent pénétrer dans la couche muqueuse épaissie et donc atteindre les bactéries présentes dans le bouchon muqueux.

liaire, bien qu'inférieure à la normale [44]. Le seul recours à ce mécanisme pour le maintien du volume du LSVA dans la mucoviscidose devient cependant particulièrement problématique quand des agressions (infections virales par exemple) viennent perturber la voie finement régulée de signalement par l'ATP. L'expression des enzymes qui hydrolysent l'ATP augmente en réponse à divers stimuli inflammatoires [46] et ce phénomène réduit le taux d'ATP et le volume du LSVA lors d'une infection expérimentale par le virus respiratoire syncytial [44] (fig. 3). Il semble donc que, dans la mucoviscidose, des agressions épisodiques des voies aériennes induisent une décompensation du système de régulation du volume du LSVA et résultent en une altération régionale de la clairance mucociliaire. Des «exacerbations» de l'atteinte respiratoire due à la mucoviscidose pourraient être l'expression clinique de ces événements épisodiques. Au cours du temps, ces agressions peuvent créer un cercle vicieux de réduction de l'élimination du mucus et d'infection bactérienne chronique, expliquant ainsi la progression chronique de cette atteinte respiratoire.

Des données en faveur de la validité de l'hypothèse «faible volume» ont été apportées par plusieurs modèles in vitro ainsi que par des mesures in vivo qui ont validé les prévisions indiquant que le LSVA était isotonique [34, 37, 47], que la mucoviscidose réduisait le volume normal du LPC $[24,34]$ et qu'une stase et une adhésion du mucus en résultaient [34]. De plus, la muqueuse du septum nasal de la souris privée du gène $C F T R$, qui est la seule région des voies aériennes présentant le phénotype de transport ionique typique de la mucoviscidose, possède un phénotype local similaire à celui de la mucoviscidose avec une réduction de la hauteur du LSVA et des signes d'infection et d'inflammation spontanées [24]. Plus récemment, une souris transgénique surexprimant la sous-unité $\beta$ de l'ENaC a été créée afin de mieux reproduire l'hyperabsorption volumi- 


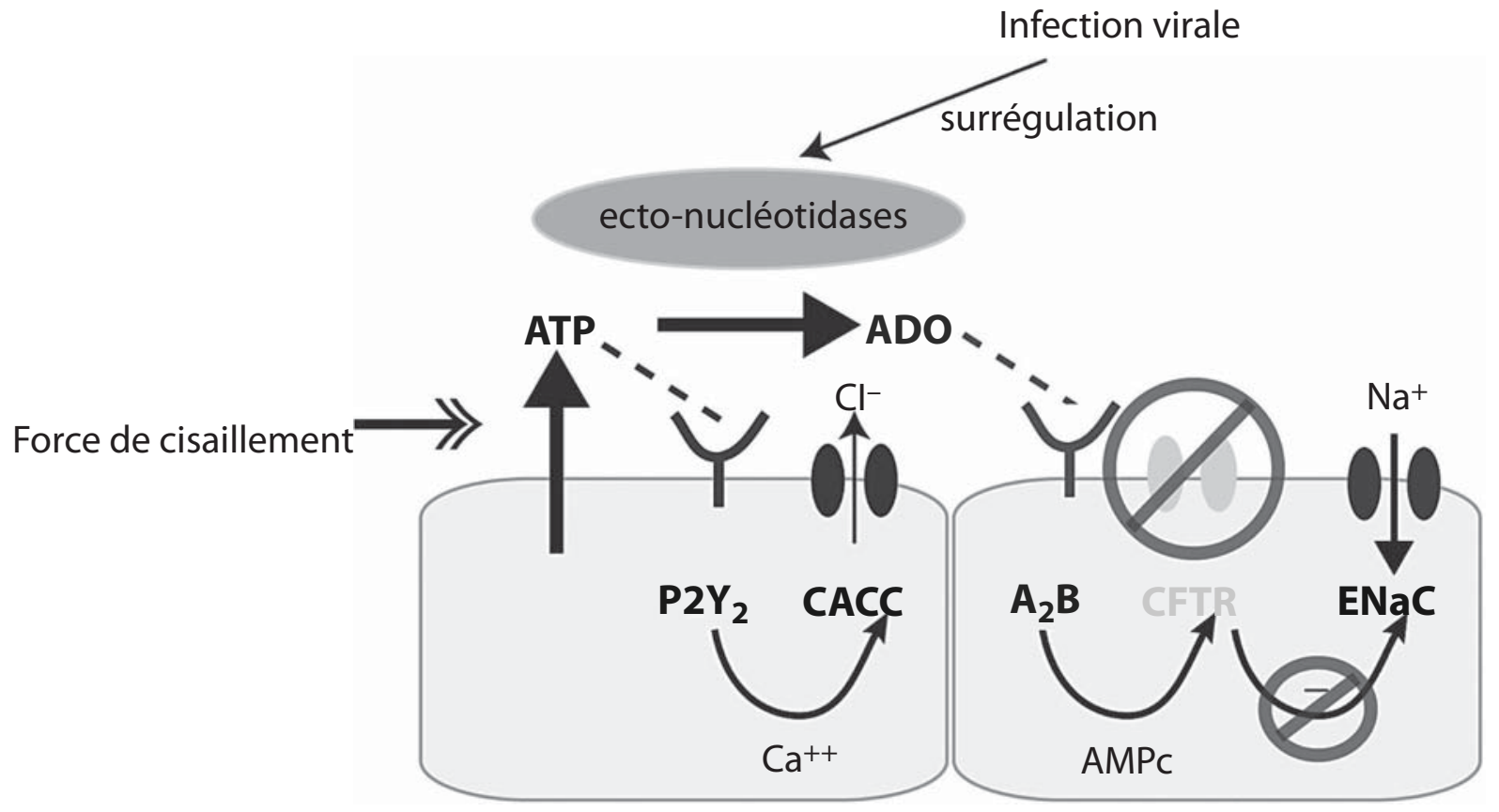

Fig. 3. L'épithélium affecté par la mucoviscidose a recours à une sécrétion de chlorure médiée par l'ATP pour la formation du LSVA. Une force de cisaillement, induite par le débit aérien sur les surfaces bronchiques, suscite une libération d'ATP, qui se lie aux récepteurs $\mathrm{P}_{2} \mathrm{Y}_{2}$ et stimule la sécrétion de chlorure par l'intermédiaire du CACC. Des ectonucléotidases dégradent l'ATP libéré, limitant son effet. Dans le contexte d'une infection virale ou d'autres stimuli inflammatoires, les ectonucléotidases sont surrégulées, le métabolisme de l'ATP s'accroît et l'aptitude à sécréter du chlorure et de l'eau (via le CACC) est altérée dans les bronches affectées par la mucoviscidose. Il faut noter que la formation d'adénosine ne stimule pas la sécrétion de chlorure et d'eau dans ces bronches en raison de l'absence de CFTR. $\mathrm{A}_{2} \mathrm{~B}=$ Récepteur $\mathrm{A}_{2} \mathrm{~B}$ de l'adénosine; $\mathrm{ADO}=$ adénosine.

que observée dans les voies aériennes humaines affectées par une mucoviscidose. Le résultat a été un animal au phénotype particulièrement proche de la mucoviscidose, avec (1) hyperabsorption démontrable de sodium a travers les voies aériennes, (2) réduction du volume du LSVA, (3) hypersécrétion de mucus et adhésion aux voies aériennes, (4) inflammation «spontanée» (ne nécessitant pas l'inoculation expérimentale d'un agent infectieux) et (5) ralentissement de l'élimination des bactéries [48].

Des mesures in vivo de la clairance mucociliaire (CMC) chez des patients atteints de mucoviscidose ont fourni d'intéressants aperçus sur la physiopathologie de l'atteinte respiratoire due à cette maladie ainsi qu'un test de l'hypothèse de la réduction de l'élimination du mucus. Le plus important ensemble de données décrivant la CMC dans la mucoviscidose est celui de Robinson et coll. [49], qui suggère que ce mécanisme est toujours anormal chez ces patients, y compris ceux dont les explorations fonctionnelles respiratoires sont normales. Cette observation permet de penser que le trouble de l'élimination du mucus est, de fait, une anomalie précoce et probablement primaire liée à la mucoviscidose et n'est pas secondaire à l'atteinte respiratoire. En revanche, des données plus récemment publiées par Donaldson et coll. [50] ont suggéré que la CMC ne différait pas de la normale dans les grosses bronches des sujets atteints de mucoviscidose, tandis que 
des anomalies de la clairance étaient détectées par des mesures traduisant l'élimination du mucus dans les petites bronches (mesures de la clairance pulmonaire périphérique et de la clairance sur 24 heures). De plus, cette étude a démontré que l'élimination de mucus médiée par la toux était négligeable à partir des bronches périphériques dans la mucoviscidose. Ces données confortent donc la notion selon laquelle l'élimination du mucus est anormale dans la mucoviscidose, mais que cette anomalie peut être hétérogène et la plus marquée dans les régions des petites bronches, où l'atteinte respiratoire paraît débuter.

\section{Progression de l'atteinte respiratoire due à la mucoviscidose - Au-delà d'une déshydratation du LSVA}

Avec la déshydratation des surfaces bronchiques dans la mucoviscidose et la formation de plaques de mucus, la scène est dressée pour la survenue d'une infection bactérienne chronique et de la réponse inflammatoire qui en résulte. Les plaques elles-mêmes, étant stagnantes et relativement impénétrables aux neutrophiles, créent une niche idéale pour des bactéries inhalées ou aspirées à partir de l'oropharynx. Tandis que du mucus s'accumule dans ces plaques, un bouchon solide peut se former et obstruer la bronche, facilitant encore l'établissement d'une infection bactérienne. Il est important de noter qu'une niche anaérobie locale de plaques et de bouchons macroscopiques de mucus se crée tant à cause d'une longue distance de diffusion de l' $\mathrm{O}_{2}$ que de la consommation élevée d' $\mathrm{O}_{2}$ par l'épithélium affecté par la mucoviscidose, comme des études in vitro et in vivo (bronchoscopie) l'ont démontré [51]. Ce microenvironnement paraît sélectionner les agents pathogènes qui lui sont le mieux adaptés, dont Pseudomonas, expliquant ainsi les observations montrant que la mucoviscidose, et, à un moindre degré, d'autres formes de bronchectasies, entraînent une vulnérabilité à un ensemble relativement restreint mais unique d'agents pathogènes typiques. Il faut noter que la complexité des communautés bactériennes qui se développent dans cet environnement est sans doute très fortement sous-estimée par les techniques microbiologiques de routine. Plus récemment, des techniques moléculaires ont permis de documenter dans le poumon mucoviscidosique une diversité bactérienne beaucoup plus importante que précédemment estimée, dont la présence de micro-organismes ne pouvant proliférer qu'en milieu anaérobie [52].

De nouvelles informations concernant le comportement des Pseudomonas dans les bronches affectées par la mucoviscidose ont été très utiles pour mieux comprendre la physiopathologie de cette maladie, et également pour permettre d'espérer la définition de nouvelles cibles thérapeutiques. Dans son environnement naturel, le Pseudomonas est une bactérie planctonique mobile. Dans les bronches atteintes par la mucoviscidose, les pseudomonadacae modifient radicalement leur expression génique, au moins en partie en raison de signaux quorum-sensing adressés en fonction de la densité bactérienne, afin qu'une microcolonie bactérienne stationnaire, ou biofilm, se développe dans les plaques et bouchons de mucus. La production d'alginate et un passage au métabolisme anaérobie, ainsi que l'environnement concentré en mucines luimême, contribuent tous au contournement des défenses immunitaires et à l'inefficacité des interventions par antibiotiques, et conduisent à la persistance des bactéries dans les bronches atteintes par la mucoviscidose. La réponse inflammatoire inefficace qui s'ensuit n'élimine pas l'infection, mais provoque au contraire des lésions bronchiques progressives et des bronchectasies.

\section{Implications thérapeutiques}

Les études des mécanismes physiopathologiques de l'atteinte respiratoire de la mucoviscidose ont non seulement conduit à mieux comprendre ce processus complexe, mais ont également permis de découvrir de nouvelles cibles thérapeutiques pour le développement de médicaments. Les normes thérapeutiques que sont la kinésithérapie respiratoire et les antibiotiques sont manifestement des moyens logiques de minimiser la masse de mucus retenu et l'obstruction bronchique tout en réduisant également la charge en bactéries infectantes qui stimulent encore l'inflammation et la production de mucus. Il apparaît cependant désormais clairement que les interventions qui restaurent l'hydratation normale du LPC et des couches muqueuses avant la survenue d'une adhésion du mucus peuvent fortement ralentir le déclenchement et la progression de l'atteinte respiratoire liée à la mucoviscidose. A cette fin, des inhalations de solutés salés hypertoniques attirant l'eau par pression osmotique dans la couche de LSVA sont maintenant employées en clinique. Cette approche représente la première génération des traitements ciblant actuellement l'anomalie sous-jacente dans la mucoviscidose. Il est gratifiant de constater que cette simple approche thérapeutique a amélioré la CMC et les explorations fonctionnelles respiratoires tout en atténuant également la symptomatologie et, surtout, en diminuant la fréquence des exacerbations [50, 53; Strandvik, p. 136]. 
D'autres agents en cours de développement visent à corriger la réduction du volume du LSVA par d'autres mécanismes d'action. Par exemple, les agonistes de $\mathrm{P}_{2} \mathrm{Y}_{2}$ de longue durée d'action (qui stimulent la sécrétion de chlorure par l'intermédiaire du CACC) et les inhibiteurs de l'ENaC font l'objet d'essais cliniques et pourraient rééquilibrer la sécrétion et l'absorption à travers les bronches mucoviscidosiques en utilisant le dispositif immédiatement disponible de transport ionique. D’autre part, d'intenses efforts utilisant le criblage à haut rendement de bibliothèques de substances chimiques débutent actuellement afin d'identifier des agents qui améliorent le traitement et la fonction cellulaires du canal CFTR le plus souvent mutant $(\Delta \mathrm{F} 508)$ afin qu'il puisse atteindre la membrane plasmatique apicale et fonctionne comme un canal chlorure [54]. Il est probable qu'il sera nécessaire d'administrer tout traitement corrigeant efficacement la réduction du volume du LSVA précocement et sans interruption (dès la petite enfance ou avant la survenue d'une atteinte respiratoire significative). Cette stratégie, pour la première fois aujourd'hui, offre toutefois l'opportunité d'intervenir de façon préventive plutôt que réactionnelle, modifiant ainsi significativement l'évolution de cette atteinte respiratoire fatale. Des essais cliniques évaluant cette approche thérapeutique chez les plus jeunes des patients sont à venir bientôt.

\section{Conclusion}

Diverses hypothèses ont été proposées afin d'expliquer l'origine de l'atteinte respiratoire due à la mucoviscidose, mais un ensemble croissant de données suggère qu'une réduction du volume du LSVA avec adhésion de mucus épaissi aux surfaces bronchiques déclenche la cascade complexe d'événements qui culminent en une infection chronique, des bronchectasies et une insuffisance respiratoire. Ces données incitent à cibler les étapes pathogènes précoces au moyen d'agents qui accroissent le volume du LSVA et détachent les plaques de mucus adhérent. De plus, les stratégies qui visent les Pseudomonas adaptés à un environnement anaérobie et à la prolifération en biofilm peuvent permettre d'améliorer le traitement des patients présentant déjà une atteinte respiratoire établie avec infection bactérienne persistante. En définitive, cependant, des traitements d'action prolongée restaurant efficacement l'hydratation des surfaces bronchiques avant la survenue de l'atteinte chronique pourront être nécessaires pour réduire for tement la charge que constitue la mucoviscidose pour celles et ceux qu'elle atteint.

\section{Références}

1 Tsui LC: Cystic fibrosis mutation database. http://www.genet.sickkids.on.ca/cftr/.

-2 di Sant' Agnese PA, Darling RC, Perera GA: Abnormal electrolyte composition of sweat in cystic fibrosis of the pancreas. Pediatrics 1953;12:549-563.

3 Quinton PM: chloride impermeability in cystic fibrosis. Nature 1983;301:421-422.

4 Knowles MR, Stutts MJ, Spock A, et al: Abnormal ion permeation through cystic fibrosis respiratory epithelium. Science 1983;221: 1067-1070.

5 Kopelman H, Durie P, Gaskin K, et al: Pancreatic fluid secretion and protein hyperconcentration in cystic fibrosis. N Engl J Med 1985;312:329-334.

6 6 Fitz JG, Basavappa S, McGill J, et al: Regulation of membrane chloride currents in rat bile duct epithelial cells. J Clin Invest 1993; 91:319-328

7 Cohn JA, Strong TV, Picciotto MR, et al: Localization of the cystic fibrosis transmembrane conductance regulator in human bile duct epithelial cells. Gastroenterology 1993; 105:1857-1864.
8 Gowen CW Jr, Gowen MA, Knowles MR: Colonic transepithelial potential difference in infants with cystic fibrosis. J Pediatr 1991; 118:412-415.

9 Riordan JR, Rommens JM, Kerem B, et al: identification of the cystic fibrosis gene: cloning and characterization of complementary DNA. Science 1989;245:1066-1073.

10 Rommens JM, Iannuzzi MC, Kerem B, et al: Identification of the cystic fibrosis gene: chromosome walking and jumping. Science 1989;245:1059-1065.

11 Bear CE, Li CH, Kartner N, et al: Purification and functional reconstitution of the cystic fibrosis transmembrane conductance regulator (CFTR). Cell 1992;68:809-818.

12 Kartner N, Hanrahan JW, Jensen TJ, et al: expression of the cystic fibrosis gene in nonepithelial invertebrate cells produces a regulated anion conductance. Cell 1991;64:681691.

13 Stutts MJ, Rossier BC, Boucher RC: Cystic fibrosis transmembrane conductance regulator inverts protein kinase A-mediated regulation of epithelial sodium channel single channel kinetics. J Biol Chem 1997;272: 14037-14040.
14 Stutts MJ, Canessa CM, Olsen JC, et al: CFTR as a CAMP-dependent regulator of sodium channels. Science 1995;269:847-850.

-15 Tarran R, Loewen ME, Paradiso AM, et al: Regulation of murine airway surface liquid volume by CFTR and $\mathrm{Ca}^{2+}$-activated $\mathrm{Cl}^{-}$ conductances. J Gen Physiol 2002;120:407418.

16 Schwiebert EM, Egan ME, Hwang TH, et al: CFTR regulates outwardly rectifying chloride channels through an autocrine mechanism involving ATP. Cell 1995;81:10631073.

17 Armstrong DS, Grimwood K, Carlin JB, et al: Lower airway inflammation in infants and young children with cystic fibrosis. Am J Respir Crit Care Med 1997;156:1197-1204.

18 Dakin CJ, Numa AH, Wang H, et al: Inflammation, infection, and pulmonary function in infants and young children with cystic fibrosis. Am J Respir Crit Care Med 2002;165: 904-910.

19 Noah TL, Black HR, Cheng PW, et al: Nasal and bronchoalveolar lavage fluid cytokines in early cystic fibrosis. J Infect Dis 1997; 175: 638-647. 
20 Muhlebach MS, Stewart PW, Leigh MW, Noah TL: Quantitation of inflammatory responses to bacteria in young cystic fibrosis and control patients. Am J Respir Crit Care Med 1999;160:186-191.

21 Khan TZ, Wagener JS, Bost T, et al: Early pulmonary inflammation in infants with cystic fibrosis. Am J Respir Crit Care Med 1995; 151:1075-1082.

22 Bonfield TL, Konstan MW, Berger M: Altered respiratory epithelial cell cytokine production in cystic fibrosis. J Allergy Clin Immunol 1999;104:72-78.

23 Tarran R, Grubb BR, Gatzy JT, et al: The relative roles of passive surface forces and active ion transport in the modulation of airway surface liquid volume and composition. J Gen Physiol 2001;118:223-236.

24 Tarran R, Grubb BR, Parsons D, et al: The CF salt controversy: in vivo observations and therapeutic approaches. Mol Cell 2001;8: 149-158.

25 Raviv U, Giasson S, Kampf N, et al: Lubrication by charged polymers. Nature 2003;425: 163-165.

26 Rhim AD, Stoykova L, Glick MC, Scanlin TF: Terminal glycosylation in cystic fibrosis (CF): a review emphasizing the airway epithelial cell. Glycoconj J 2001;18:649-659.

27 Scanlin TF, Glick MC: Terminal glycosylation in cystic fibrosis. Biochim Biophys Acta 1999;1455:241-253.

-28 Bryan R, Kube D, Perez A, et al: Overproduction of the CFTR R domain leads to increased levels of asialoGM1 and increased Pseudomonas aeruginosa binding by epithelial cells. Am J Respir Cell Mol Biol 1998;19:269-277.

29 Pier GB, Grout M, Zaidi TS, et al: Role of mutant CFTR in hypersusceptibility of cystic fibrosis patients to lung infections. Science 1996;271:64-67.

-30 Schroeder TH, Reiniger N, Meluleni G, et al: Transgenic cystic fibrosis mice exhibit reduced early clearance of pseudomonas aeruginosa from the respiratory tract. J Immunol 2001;166:7410-7418.

-31 Zar H, Saiman L, Quittell L, Prince A: Binding of Pseudomonas aeruginosa to respiratory epithelial cells from patients with various mutations in the cystic fibrosis transmembrane regulator. J Pediatr 1995; $126: 230-233$
32 Smith JJ, Travis SM, Greenberg EP, Welsh MJ: Cystic fibrosis airway epithelia fail to kill bacteria because of abnormal airway surface fluid. Cell 1996;85:229-236.

- 33 Goldman MJ, Anderson GM, Stolzenberg ED, et al: Human beta-defensin-1 is a saltsensitive antibiotic in lung that is inactivated in cystic fibrosis. Cell 1997;88:553-560.

34 Matsui H, Grubb BR, Tarran R, et al: Evidence for periciliary liquid layer depletion, not abnormal ion composition, in the pathogenesis of cystic fibrosis airways disease. Cell 1998;95:1005-1015.

35 Grubb BR, Chadburn JL, Boucher RC: In vivo microdialysis for determination of nasal liquid ion composition. Am J Physiol Cell Physiol 2002;282:C1423-C1431.

-36 Knowles MR, Robinson JM, Wood RE, et al: Ion composition of airway surface liquid of patients with cystic fibrosis as compared with normal and disease-control subjects. J Clin Invest 1997;100:2588-2595.

37 Jayaraman S, Song YL, Vetrivel L, et al: Noninvasive in vivo fluorescence measurement of airway-surface liquid depth, salt concentration, and pH. J Clin Invest 2001;107:317324.

38 Caldwell RA, Grubb BR, Tarran R, et al: In vivo airway surface liquid $\mathrm{Cl}$ - analysis with solid-state electrodes. J Gen Physiol 2002; 119:3-14.

-39 Travis SM, Conway BA, Zabner J, et al: Activity of abundant antimicrobials of the human airway. Am J Respir Cell Mol Biol 1999; 20:872-879.

40 Tate S, MacGregor G, Davis M, et al: Airways in cystic fibrosis are acidified: detection by exhaled breath condensate. Thorax 2002;57: 926-929.

41 Song Y, Salinas D, Nielson DW, Verkman AS: Hyperacidity of secreted fluid from submucosal glands in early cystic fibrosis. Am J Physiol Cell Physiol 2006;290:C741-C749.

42 Coakley RD, Grubb BR, Paradiso AM, et al: Abnormal surface liquid $\mathrm{pH}$ regulation by cultured cystic fibrosis bronchial epithelium. Proc Natl Acad Sci USA 2003;100: 16083-16088

43 Lazarowski ER, Tarran R, Grubb BR, Van Heusden CA, et al: Nucleotide release provides a mechanism for airway surface liquid homeostasis. J Biol Chem 2004;279:3685536864.
44 Tarran R, Button B, Picher M, et al: Normal and cystic fibrosis airway surface liquid homeostasis: the effects of phasic shear stress and viral infections. J Biol Chem 2005;280: 35751-35759.

45 Matsui H, Verghese MW, Kesimer M, et al: Reduced three-dimensional motility in dehydrated airway mucus prevents neutrophil capture and killing bacteria on airway epithelial surfaces. J Immunol 2005;175:10901099.

46 Picher M, Burch LH, Boucher RC: Metabolism of $\mathrm{P} 2$ receptor agonists in human airways: implications for mucociliary clearance and cystic fibrosis. J Biol Chem 2004;279: 20234-20241.

-47 Jayaraman S, Song YL, Verkman AS: Airway surface liquid osmolality measured using fluorophore-encapsulated liposomes. J Gen Physiol 2001;117:423-430.

48 Mall M, Grubb BR, Harkema JR, et al: Increased airway epithelial $\mathrm{Na}^{+}$absorption produces cystic fibrosis-like lung disease in mice. Nat Med 2004;10:487-493.

49 Robinson M, Eberl S, Tomlinson C, et al: Regional mucociliary clearance in patients with cystic fibrosis. J Aerosol Med 2000;13: 73-86.

50 Donaldson SH, Bennett WD, Zeman KL, et al: Mucus clearance and lung function in cystic fibrosis with hypertonic saline. N Engl J Med 2006;354:241-250.

51 Worlitzsch D, Tarran R, Ulrich M, et al: Effects of reduced mucus oxygen concentration in airway pseudomonas infections of cystic fibrosis patients. J Clin Invest 2002; 109:317-325.

-52 Rogers GB, Carroll MP, Serisier DJ, et al: Characterization of bacterial community diversity in cystic fibrosis lung infections by use of 16s ribosomal DNA terminal restriction fragment length polymorphism profiling. J Clin Microbiol 2004;42:5176-5183.

53 Elkins MR, Robinson M, Rose BR, et al: A controlled trial of long-term inhaled hypertonic saline in patients with cystic fibrosis. $\mathrm{N}$ Engl J Med 2006;354:229-240.

54 Pedemonte N, Lukacs GL, Du K, et al: Smallmolecule correctors of defective deltaF508CFTR cellular processing identified by highthroughput screening. J Clin Invest 2005; 115:2564-2571. 